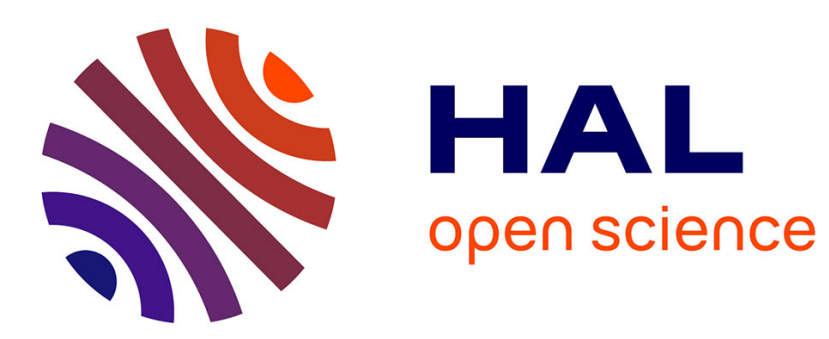

\title{
A stepwise method for identification of controlled discrete manufacturing systems
}

Ana Paula Estrada-Vargas, Jean-Jacques Lesage, Ernesto López-Mellado

\section{To cite this version:}

Ana Paula Estrada-Vargas, Jean-Jacques Lesage, Ernesto López-Mellado. A stepwise method for identification of controlled discrete manufacturing systems. International Journal of Computer Integrated Manufacturing, 2015, 28 (2), pp.187 - 199. 10.1080/0951192X.2013.874591 . hal-01088640

\section{HAL Id: hal-01088640 https://hal.science/hal-01088640}

Submitted on 28 Nov 2014

HAL is a multi-disciplinary open access archive for the deposit and dissemination of scientific research documents, whether they are published or not. The documents may come from teaching and research institutions in France or abroad, or from public or private research centers.
L'archive ouverte pluridisciplinaire HAL, est destinée au dépôt et à la diffusion de documents scientifiques de niveau recherche, publiés ou non, émanant des établissements d'enseignement et de recherche français ou étrangers, des laboratoires publics ou privés. 


\title{
A Stepwise Method for Identification of Controlled Discrete Manufacturing Systems
}

\author{
Ana Paula Estrada-Vargas ${ }^{1,2}$, Jean-Jacques Lesage ${ }^{2}$, Ernesto López-Mellado ${ }^{1 *}$ \\ ${ }^{1}$ CINVESTAV Unidad Guadalajara. Av. del Bosque 1145. Col. El Bajío 45015 Zapopan, Mexico \\ ${ }^{2}$ LURPA Ecole Normale Supérieure de Cachan. 61, Av. du Président Wilson. 94235 Cachan Cedex, France \\ \{aestrada, elopez \}@gdl.cinvestav.mx, Jean-Jacques.lesage@lurpa.ens-cachan \\ *Corresponding author
}

\begin{abstract}
This paper deals with the identification of discrete event manufacturing systems that are automated by a programmable logic controller (PLC). The behaviour of the closed loop system (PLC and Plant) is observed during its operation and is represented by a single long sequence of observed input/output signals vectors. The proposed method follows a black-box and passive identification approach; it allows building stepwise an interpreted Petri net (IPN) model. The identification method is composed of several polynomial time algorithms implemented in a software tool that generates and draws the IPN model.
\end{abstract}

Keywords: Controlled manufacturing system, DES; Stepwise identification; Interpreted Petri nets; Automated modelling

\section{Introduction}

Identification of discrete event systems (DES) from external observation of the system behaviour has interesting applications such as reverse engineering for (partially) unknown systems, fault diagnosis, or formal verification. Analogously to continuous identification techniques, identification methods for DES yield a mathematical model that represents the observed behaviour and closely approximates the actual DES' behaviour. The interest in obtaining a model of an existing system is that in industrial processes under intensive workload, initial models are not always updated after (software) maintenance (Lanubile, 2002) leading to documentation not aligned with the current system operation.

\subsection{Related works}

Methods for identifying DES have been proposed, firstly in the computer science community as techniques for representing languages, and later in automation as techniques for discovering the functioning of discrete manufacturing processes.

In the first methods, considered as learning techniques (Gold, 1967) (Angluin, 1988), the problem is dealt as of obtaining a language representation from sets of accepted words; such methods are considered as learning techniques. Several works adopted diverse classes of state machines as representation model (Kella, 1971) (Richetin, 1984), others use context free grammars; Petri nets (PN) models are also used for sequence descriptions (Hirahishi, 1992).

In recent years, the proposed identification methods aim the construction of models (Petri nets or automata) describing the functioning of DES, whose behaviour is unknown or ill-known, from the observation of the exhibited behaviour in the form of sequences of activities and/or events. In the context of industrial automated manufacturing systems, identification methods allow obtaining an approximated model that can be detailed using established modelling techniques and available knowledge of the system; such a model describes the controller/plant behaviour during the closed- 
loop functioning. Three main classes of approaches for identifying DES have been proposed in literature (Estrada, 2010a).

The incremental synthesis approach, proposed in (Meda, 2001, 2003, 2005) deals with unknown partially observable DES exhibiting cyclic behaviour. Several algorithms have been proposed allowing the on-line identification of concurrent DES from output sequences. Although the techniques are efficient, the obtained interpreted PN (IPN) models may represent more sequences than those observed and system's inputs are not taken into account.

Another recent method (Klein, 2005) allows building efficiently a non deterministic finite automaton (NDFA) from a set of input/output sequences, experimentally measured from the DES to be identified. Under several hypotheses, the constructed NDFA generates exactly the same input/output (I/O) sequences of given length than observed ones. The method was conceived for fault detection in a model-based approach (Roth, 2009). Extensions to this work propose an identification method performing optimal partitioning of concurrent subsystems for distributed fault detection purposes (Roth, 2010).

The techniques based on integer linear programming (ILP) approach lead to free-labelled Petri net models representing exactly the observed behaviour in the form of event sequences (Giua, 2005). However both the ILP problem statement from event sequences and the processing have exponential complexity. This approach is held in several extensions to the first work (Cabasino, 2007), (Dotoli, 2008, 2011), (Fanti, 2008); nevertheless, issues regarding applications to actual industrial DES have not yet been addressed in these works.

Other works on the matter, based on different approaches have been proposed. The techniques for workflow mining, published by van der Aalst and co-authors (Cook, 2004), (van der Aalst, 2004), allows building Petri net models of workflow processes in which all the activities are observable. Other works pursue the construction of a stochastic PN from recorded event sequences (Ould El Medhi, 2006, 2012) for reliability applications.

\subsection{Input-output approach}

In this paper the problem of identifying a DES controlled by a PLC during its operation is addressed. Both controller's inputs and outputs are sampled for building a single long sequence of $\mathrm{I} / \mathrm{O}$ vectors which are stored in a database (figure 1); afterwards it is processed off line, yielding an IPN model. Petri nets have been held in this research for their power of expression of complex behaviours exhibited by discrete manufacturing systems (Dicesare, 1993), (Jeng, 2003) (Gradisar, 2007).

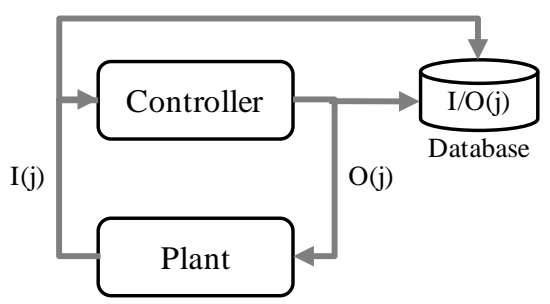

Figure 1. Closed loop controller-plant DES

In order to illustrate our motivation, consider a simple DES with three input signals (generated by sensors: a, b, c) and three output signals (for the control of pre-actuators: A, B, C), whose binary 
I/O vectors have the following correspondence: $\left[\begin{array}{llllllll}a & b & c & \mid & A & B & C\end{array}\right]^{T}$. If the following short I/O vector sequence $w$ is observed and provided to our identification procedure

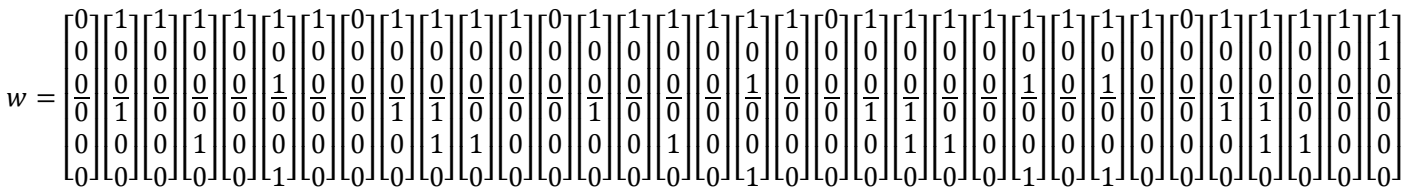

the IPN model shown in figure 2 is obtained, which reproduces the observed sequence.

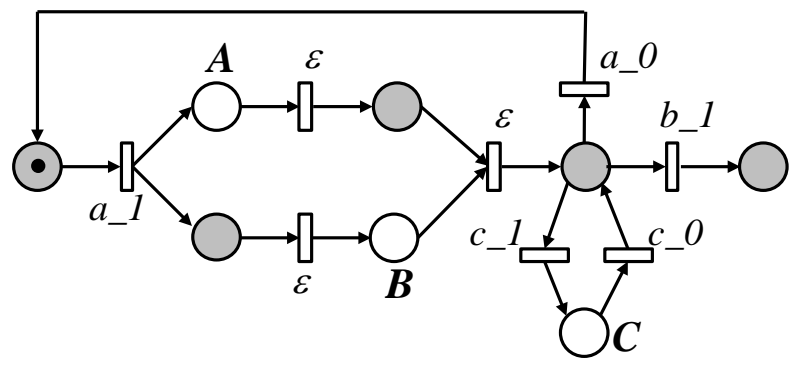

Figure 2. An IPN model representing the sequence w

The method proposed herein provides a solution to the problem informally stated in the above example. It is based on a previously published method for the identification of concurrent partially observable DES (Estrada 2010b) that allow processing a set of cyclic input/output sequences and yields IPN models including silent transitions and non-labelled places. Nevertheless, the assumption regarding the input/output sequences to be cyclic is not always fulfilled in manufacturing systems. Furthermore, this assumption does not allow identifying non repetitive behaviours, such as initialising sequences.

\subsection{Contribution}

This identification technique has afterwards been extended and adapted for PLC-based controlled discrete manufacturing systems, which operate during a long time period performing repetitive tasks (Estrada 2011). In this paper, the assumption that cyclic sequences are given as input data is dropped off by considering a single I/O sequence that captures both the transient and the repetitive behaviour. This new technique allows detecting the states in which the system iterates for executing repetitive tasks, based on the notion of $\kappa$-equivalence, allowing building progressively a safe IPN. Furthermore, this feature allows updating the model when new observed input/output vectors are added to the sequence. In the present paper the results in (Estrada 2011) are recalled and the new approach coping with non-cyclic behaviour is presented in detail; furthermore, a software tool that implements the proposed algorithms has been developed for automating the model construction.

The paper is organized as follows. In section 2 the background and useful notations on Petri nets and languages are recalled. In section 3, the problem of identifying industrial processes is stated and the stepwise method is presented. In section 4 a case study is dealt thanks to a software tool implementing the proposed method.

\section{Preliminaries}

This section presents the basic concepts and notation of Petri Nets (PN) and Interpreted Petri nets (IPN) used in this paper. 
Definition 1: An ordinary Petri Net structure $G$ is a bipartite digraph represented by the 4-tuple $G=(P, T$, Pre, Post $)$ where: $P=\left\{p_{1}, p_{2}, \ldots, p_{|P|}\right\}$ and $T=\left\{t_{1}, t_{2}, \ldots, t_{|T|}\right\}$ are finite sets of vertices named places and transitions respectively; Pre (Post): $P \times T \rightarrow\{0,1\}$ is a function representing the arcs going from places to transitions (from transitions to places). Usually, places are depicted as circles and transitions as bars or rectangles; arcs are depicted as arrows.

The symbol $t_{j}\left(t_{j}{ }^{\prime}\right)$ denotes the set of all places $p_{i}$ such that $\operatorname{Pre}\left(p_{i}, t_{j}\right) \neq 0\left(\operatorname{Post}\left(p_{i}, t_{j}\right) \neq 0\right)$. Such places are called input (output) places of $t_{j}$. Analogously, ${ }^{\circ} p_{i}\left(p_{i}{ }^{\circ}\right)$ denotes the set of input (output) transitions of $p_{i}$.

The incidence matrix of $G$ is $C=C^{+}-C^{-}$, where $C^{-}=\left[c_{i j}{ }^{-}\right] ; c_{i j}{ }^{-}=\operatorname{Pre}\left(p_{i}, t_{j}\right)$; and $C^{+}=\left[c_{i j}{ }^{+}\right]$; $c_{i j}{ }^{+}=\operatorname{Post}\left(p_{i}, t_{j}\right)$ are the pre-incidence and post-incidence matrices respectively.

A marking function $M: P \rightarrow \mathbb{Z}^{+}$represents the number of tokens (depicted as dots) residing inside each place; it is usually expressed as an $|P|$-entry vector. $\mathbb{Z}^{+}$is the set of nonnegative integers.

Definition 2: A Petri Net system or Petri Net $(P N)$ is the pair $N=\left(G, M_{0}\right)$, where $G$ is a $P N$ structure and $M_{0}$ is an initial marking.

In a PN system, a transition $t_{j}$ is enabled at marking $M_{k}$ if $\forall p_{i} \in P, M_{k}\left(p_{i}\right) \geq \operatorname{Pre}\left(p_{i}, t_{j}\right)$; an enabled transition $t_{j}$ can be fired reaching a new marking $M_{k+1}$ which can be computed as $M_{k+1}=M_{k}+C v_{k}$, where $v_{k}(i)=0, \mathrm{i} \neq \mathrm{j}, v_{k}(\mathrm{j})=1$, this equation is called the PN state equation. The reachability set of a PN is the set of all possible reachable markings from $M_{0}$ firing only enabled transitions; this set is denoted by $R\left(G, M_{0}\right)$.

A PN is called safe if $\forall M_{k} \in R\left(G, M_{0}\right), \forall p_{i} \in P, M_{k}\left(p_{i}\right) \leq 1$.

Now it is defined IPN, an extension to $P N$ that allows associating input and output signals to PN models.

Definition 3 : An IPN $\left(Q, M_{0}\right)$ is a net structure $Q=(G, \Sigma, \Phi, \lambda, \varphi)$ with an initial marking $M_{0}$ where:

$G$ is a PN structure, $\Sigma=\left\{\alpha_{1}, \alpha_{2}, \ldots, \alpha_{r}\right\}$ is the input alphabet, and $\Phi=\left\{\phi_{1}, \phi_{2}, \ldots, \phi_{q}\right\}$ is the output alphabet.

$\lambda: T \rightarrow \Sigma \cup\{\varepsilon\}$ is a labelling function of transitions, where $\varepsilon$ represents a system internal event that is externally uncontrollable; the symbol $\varepsilon$ must not be associated to more than one $t_{j} \in p_{i}{ }^{*}$.

$\varphi: R\left(Q, M_{0}\right) \rightarrow\left(\mathbb{Z}^{+}\right)^{q}$ is an output function, that associates to each marking in $R\left(Q, M_{0}\right)$ a $q$-entry output vector; $q=|\Phi|$ is the number of outputs. $\varphi$ is represented by a $q \times|P|$ matrix, such that if the output symbol $\phi_{i}$ is present (turned on) every time that $M\left(p_{j}\right) \geq 1$, then $\varphi(i, j)=1$, otherwise $\varphi(i, j)=0$.

When an enabled transition $t_{j}$ is fired in a marking $M_{k}$, then a new marking $M_{k+1}$ is reached. This

behaviour is represented as $M_{k} \stackrel{t_{j}}{\longrightarrow} M_{\mathrm{k}+1}$; the state equation is completed with the marking projection $y_{k}=\varphi M_{k}$, where $y_{k} \in\left(\mathbb{Z}^{+}\right)^{q}$ is the $k$-th output vector of the IPN.

According to functions $\lambda$ and $\varphi$, transitions and places of an IPN $\left(Q, M_{0}\right)$ can be classified as follows.

Definition 4: If $\lambda\left(t_{i}\right) \neq \varepsilon$ the transition $t_{i}$ is said to be controllable ( $t_{\mathrm{i}}$ can be fired when the associated input symbol is present). Otherwise it is uncontrollable ( $t_{\mathrm{i}}$ is autonomously fired). A place $p_{i} \in P$ is said to be measurable if the $i$-th column vector of $\varphi$ is not null, i.e. $\varphi(\bullet, i) \neq 0$. 
Otherwise it is non-measurable. $P=P^{m} \cup P^{u}$ where $P^{m}$ is the set of measurable places and $P^{u}$ is the set of non-measurable places.

Definition 5: The l-length I/O language $\mathcal{L}\left(Q, M_{0}\right)$ of an $I P N\left(Q, M_{0}\right)$ contains words of length $l$ formed by pairs $\left(\lambda\left(t_{k}\right), y_{k}\right)$, where $y_{k}=\varphi M_{k}$.

$$
\begin{aligned}
\mathcal{L}\left(Q, M_{0}\right)=\left\{\left(\lambda\left(t_{i+1}\right), \varphi\left(M_{i+1}\right)\right)\left(\lambda\left(t_{i+2}\right), \varphi\left(M_{i+2}\right)\right) \ldots\left(\lambda\left(t_{i+1}\right), \varphi\left(M_{i+1}\right)\right)\right. \\
\left.\mid M_{i} \stackrel{t_{i+1}}{\longrightarrow} M_{i+2} \ldots \stackrel{t_{i+1}}{\longrightarrow} M_{i+l} ; M_{i} \in R\left(Q, M_{0}\right)\right\}
\end{aligned}
$$

\section{Stepwise Identification}

\subsection{Problem statement}

Consider a DES composed of a Plant and a Controller (a PLC) operating in a closed-loop as shown in Figure 1. It is assumed that the data exchanged between Plant and PLC consist of binary signals. The input signals of the PLC (outputs of the Plant) are generated by the sensors of the Plant. The output signals of the PLC (inputs of the Plant) control the actuators of the Plant. The external behaviour of such a DES can be observed (and characterized) by the change of values of all input/output (I/O) signals exchanged between the controller and the plant.

The signal values of all inputs and outputs at a given instant $j$ can be arranged to form an $\mathrm{I} / \mathrm{O}$ vector $\mathrm{I} / \mathrm{O}(\mathrm{j})=\left[\alpha_{1}(\mathrm{j}) \alpha_{2}(\mathrm{j}) \ldots \alpha_{r}(\mathrm{j}) \mid \phi_{1}(\mathrm{j}) \phi_{2}(\mathrm{j}) \ldots \phi_{q}(\mathrm{j})\right]^{\mathrm{T}}$.

At the end of every PLC cycle, the current value of all Inputs and Outputs can be easily captured and recorded in a data base. Each new observed I/O vector (when at least one I/O changes its value) belongs to an $\mathrm{I} / \mathrm{O}$ vector alphabet.

Definition 6: The I/O vector alphabet of a DES $S$ with $r$ inputs and q outputs is $\Sigma_{I / O} \subseteq\{1,0\}^{(r+q)}$. A vector represents a configuration of I/O binary values.

The concatenation of I/O vectors observed consecutively forms a sequence $w$, which is the input data of the identification algorithm.

Definition 7: The observed input-output sequence of a DES with $r$ inputs and $q$ outputs is: $w=w(1) w(2) \ldots w(j) \ldots$, where $w(j)=[I(j) O(j)]^{T}$ is the j-th observed $\mathrm{I} / \mathrm{O}$ vector belonging to $\Sigma_{I / O}$.

The observed language of $S$ is a singleton $\mathcal{L}(S)=\{w\}$, which can be decomposed into substrings of length equal or less than $l$. The language of all these substrings is defined below.

Definition 8: The observed input-output language of length $l$ of a DES $S$ is defined as $\boldsymbol{L}^{l}(S)=$ $\{\varepsilon\} \cup\{w(i+1) w(i+2) \ldots w(i+h) \mid 1 \leq i+h \leq l\}$.

Now the identification problem can be formulated as follows: given a DES whose only available information is a single observed I/O sequence $w$ arbitrarily large and an accuracy parameter $\kappa$, the aim of the identification process is to obtain a safe IPN model $\left(Q, M_{0}\right)$ such that $\mathcal{L}_{\text {out }}^{\kappa}\left(Q, M_{0}\right)=$ $\mathcal{L}^{\kappa}(S)$. The parameter $\kappa$ is used to adjust the accuracy of the identified model, similarly as proposed in (Klein, 2005).

It is important to point out that the aim of obtaining a model through identification is not to represent exactly the observed language, but to represent the observed behaviour and to infer actual behaviour that has not been observed during data collection. In order to perform this inference, the parameter $\kappa$, is used as an indicator for measuring the state equivalence. When $\kappa$-equivalent states are found, they are merged, increasing the accepted language of the IPN and consequently the modelled behaviour. The notion of state equivalence with respect to $\kappa$ is explained below. 


\subsection{Outline of the strategy}

The method allows the progressive construction of a safe IPN representing exactly the sampled input/output language of length $\kappa+1$ of the DES.

From the I/O vector sequence, an event sequence is computed and a sequence of event substrings of length $\kappa$ is built. Every substring is associated to a transition of a PN, which describes the causal relationship between event substrings. A PN node path formed by non-measurable places represents the substring sequence; this path is built taking into account the possible repetitive observed behaviour (internal model). Then simplifications rules may be applied. Notice that the number of non-observable places is not predefined.

Finally, the model is completed by including observable places which are related to pertinent transitions in the PN according to output changes provoked by events; input symbols are also associated to transitions. This part of the algorithm can be concurrently performed at any moment, for example when a cycle is identified, whilst the internal model is updated by processing the new $\mathrm{I} / \mathrm{O}$ vectors.

\section{3. $\mathrm{I} / \mathrm{O}$ sequence processing}

\subsubsection{Events sequence}

The I/O vector sequence $w$ is progressively built by adding new observed I/O vectors. Variations in any of input or output between two consecutive I/O vectors in $w$ are considered as events, which are relevant in the identification method, in which a string of observed events are computed (Estrada 2010a).

Definition 9: An observed event vector $\tau(j)$ is the variation between two consecutive I/O vectors: $\tau(j)=w(j+1)-w(j)$. The $r$ first entries of $\tau(j)$, denoted as $\beta(\tau(j))$ correspond to the variation between two consecutive input vectors $I(j), I(j+1)$ (input event).

Events can be represented in a more compact way. A symbolic input event $\lambda^{\prime}(\tau(j))$ is a string representation of the input event vector $\beta(\tau(j))$. $I_{i} \_1$ denotes the change from 0 to 1 of the input $I_{i}$; similarly, $I_{i} 0$ denotes the change from 1 to 0 of the input $I_{i} \cdot \lambda^{\prime}(\tau(j))$ is computed as:

$$
\lambda^{\prime}\left(\tau_{i}(j)\right)=\prod_{i=1}^{m} I_{i-} X \quad \text { where } I_{i_{-}} x=\left\{\begin{array}{ccc}
I_{i-1} & \text { if } & I_{i}(j+1)-I_{i}(j)=1 \\
I_{i-} 0 & \text { if } & I_{i}(j+1)-I_{i}(j)=-1 \\
\varepsilon & \text { if } & I_{i}(j+1)-I_{i}(j)=0
\end{array}\right.
$$

Then for a sequence $w$, a sequence of observed events $\tau(j)=\tau(1) \tau(2) \ldots \tau(j) \ldots$ is computed. During the process, if the difference between two consecutive I/O vectors has not been observed before, a new event $e_{j}$ is created $\left(\tau(j)=e_{j}\right)$. The maximum number of possible event vectors is $3^{(r+q)}-1$. However, in practice, only a small subset of them is observed because the plant and the controller are mutually constraining their behaviour.

\subsection{2. $\kappa$-length event traces}

Events $e_{j}$ represent changes in the observable behaviour of the system. However several identical events may be generated during different internal states during the system execution. In order to distinguish within the internal behaviour when these changes are exhibited, $\kappa$ precedent events are considered. The notion of $\kappa$-length event trace is used to distinguish such situations.

Definition 10: An event trace $\tau^{k}(j)$ is the substring from $\tau$ of length $\kappa$ whose last event is $\tau^{k}(j)$. $\tau^{\kappa}(j)=\tau(j-\kappa+1) \tau(j-\kappa+2) \ldots \tau(j)$. 
This notion is useful to determine during the identification process if two states represent the same internal behaviour. For this puepose, the notion of equivalent states involving the history of previous $\kappa$ events is used.

Definition 11: Two states of the model representing the identified system are $\kappa$-equivalent if the event traces $\tau^{k}(j)$ leading to such states are the same.

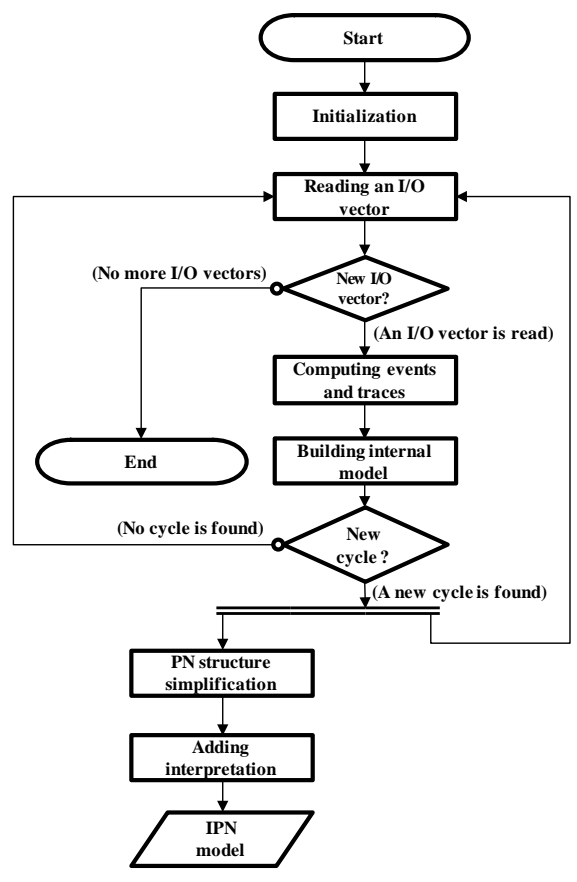

Figure 3. Stages of the identification algorithm

\subsection{Identification algorithm}

The procedure for building the IPN model from the I/O sequence can be summarized in the block diagram of Figure 3. It consists of five main steps that are described below.

Step 1. Initialization

In this step, a PN structure is initiated. This is done by executing the following statements:

$T \leftarrow \varnothing ; E T \leftarrow \varnothing ; P \leftarrow\left\{p_{\text {ini }}\right\}$; //Create an initial empty set of transitions $\mathrm{T}$, an initial empty event traces set ET, and an initial set of places $P$ containing a place $p_{\text {ini }}$

$M_{0}\left(p_{\text {ini }}\right) \leftarrow 1 ; \mu\left(p_{\text {ini }}\right) \leftarrow w(1)$; current $\leftarrow p_{\text {ini }}$; //Put a token on $p_{\text {ini }}$ and associate to it the first observed vector w(1). Take such a place as current.

Step 2. Building the events and traces

Once the net is initiated, the procedure iterates on new $\mathrm{I} / \mathrm{O}$ vectors in $w$. When an input or an output changes its value, an $\mathrm{I} / \mathrm{O}$ vector is considered to update the events sequence and the events traces according to Definition 9 and Definition 10 respectively.

Step 3. Building the internal model

Let $e_{j}$ be the last event in the trace $\tau^{\kappa}(j)$; the associated transition will be denoted as $t_{r}^{e_{j}}$ (several transitions may have associated the same $e_{j}$ ). The internal model, composed of non observable places can be systematically built following the next procedure. 
Algorithm 1. Internal model construction

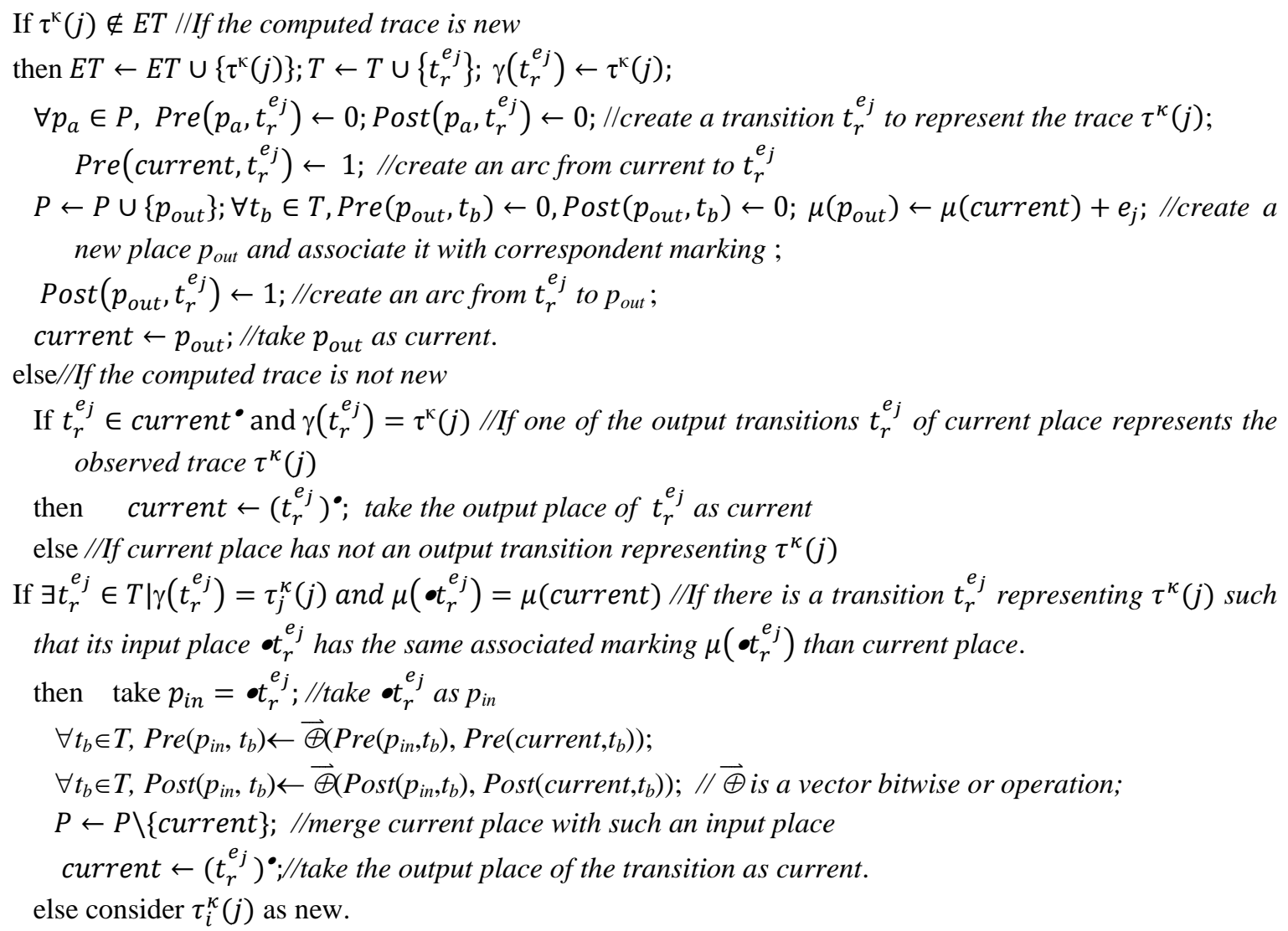

Step 4. PN structure simplification

After performing step 3, the algorithm waits for an I/O change by returning to step 2. Nevertheless, notice that merging places through step 3 of the algorithm could lead to merging of equivalent transitions. When such a merging is performed, a cycle on the PN is created. This is considered as a representative change in the structure of the model, and thus, simultaneously with launching of step 2, step 4 is executed to make a PN simplification procedure. Such a procedure based on concurrence transformations has already been explained in (Estrada 2009). It basically consists of the analysis of different paths between two places containing transition permutations leading to concurrent components transformations. If there exist $m$ ! paths, it is verified if every one of them is a permutation from each other, and the subnet can be transformed into a concurrent component of G' preserving the same behaviour.

Step 5. Adding interpretation

Once the PN has been simplified through step 4, input and output information is included on the model, obtaining an IPN representing the language $\mathcal{L}^{\kappa}(S)$ of the DES that has been observed. Input information is added to the IPN by associating symbols to transitions according to the symbolic event input function of Definition 9. The procedures to add output information and simplify implicit non observable places are summarised below and deeply explained in (Estrada, 2009). 
Algorithm 2. Representing outputs in the internal model

1. Create $q$ measurable places corresponding to each one of the outputs of the system.

2. Add arcs to and from the measurable places to the transitions of the net, according to its associated event $e_{j}$.

3. Put tokens in the corresponding measurable places to represent the first observed output vector.

4. If there is a non-measurable place whose input and output transitions are exactly the same than any measurable place, remove such a non-measurable place and its input and output arcs.

\subsection{Complexity of the identification algorithm}

First the complexity of each of the steps of the algorithm is analysed.

- Step 1 consists of a reduced number of initialising operations; it can be considered as $\Theta(c)$ where c is a constant.

- Step 2 computes the events sequence from $w$; the difference between two consecutive I/O vectors requires performing $r+q$ operations; thus the total number of operations is $(|w|-1)(r+q)$. Considering that the number of inputs and output signals is small compared to the length of $w$, the complexity can be approximated to $\Theta(|w|)$.

- Step 3 computes and processes the event traces: the computation of traces implies to perform $\Theta(|w|)$ iterations; the processing of traces involves a search with help of a hash table, thus the number of operations is $\Theta(c)$. If the trace is found, corresponding operations for merging pertinent places depend on the number of input and output transitions of such places that may also be considered as small; thus we can consider the merging operations are $\Theta(c)$. If the trace is not found, the number of operations for adding a new transition and place is $(|w|+|w|-1)$ in the worst case, that is, approximated by $\mathrm{O}(|w|)$. However, if there are cycles in the system, the number of places and transitions added decrease and they may be very small with respect to $v$. Thus, the whole step is, in the worst case, $\mathrm{O}\left(v^{2}\right)$.

- Step 4 may involve the analysis of $m$ ! paths in a PN substructure, where $m$ is number of possible concurrent transitions. However, this number usually small; thus it can be considered as $\Theta$ (c).

- Step 5 associates symbolic events to the created transitions, whose number in the worst case is $v$; in this step $q$ places are associated to transitions; thus the complexity is $\mathrm{O}(|w|)$.

The highest complexity is due to the step 3 , which builds the internal model; thus the computational complexity of the identification procedure is $\mathrm{O}\left(|w|^{2}\right)$.

\subsection{Example 1}

Consider again the DES outlined in the introduction; it has three output signals, $\Phi=\{A, B, C\}$, and three input signals $\Sigma=\{\mathrm{a}, \mathrm{b}, \mathrm{c}\}$. The entries of the binary $\mathrm{I} / \mathrm{O}$ vectors have the following correspondence: $\left[\begin{array}{llllllll}a & b & c & \mid & A & B & C\end{array}\right]^{T}$. An I/O sequence is progressively observed. The first measured $\mathrm{I} / \mathrm{O}$ vector (which could correspond to the initial state) of the DES is: $w(1)=\left[\begin{array}{lllllll}0 & 0 & 0 & \mid & 0 & 0 & 0\end{array}\right]^{T}$.

When a second I/O vector $w(2)=\left[\begin{array}{lllllll}1 & 0 & 0 & \mid & 1 & 0 & 0\end{array}\right]^{T}$ is processed, the event vector $\tau(1)=$ $e_{1}=\left[\begin{array}{lllllll}1 & 0 & 0 & \mid & 1 & 0 & 0\end{array}\right]^{T}$ is computed; the input event vector is $\lambda(1)=\left[\begin{array}{lll}1 & 0 & 0\end{array}\right]^{T}$ and its corresponding symbolic input event is $\lambda^{\prime}(1)=a_{-} \_$, i.e. the rising edge of a. 
Considering a value $\kappa=1$, we can compute the first event trace $\tau^{1}(1)=e_{1}$. Notice that, in this case, trace and event are the same. This event trace is related with a transition of the IPN. The IPN constructed after observing two I/O vectors is shown in Figure 4.

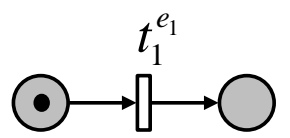

Figure 4. PN representing $e_{1}$

When a third $\mathrm{I} / \mathrm{O}$ vector $w(3)=\left[\begin{array}{lllllll}1 & 0 & 0 & \mid & 0 & 0 & 0\end{array}\right]^{T}$ is processed, $\tau(2)=e_{2}=$ $\left[\begin{array}{lllllll}0 & 0 & 0 & \mid & -1 & 0 & 0\end{array}\right]^{T}, \lambda(2)=\left[\begin{array}{lll}0 & 0 & 0\end{array}\right]^{T}$ and $\lambda^{\prime}(2)=\varepsilon$ are computed and the model is updated, as shown in Figure 5.

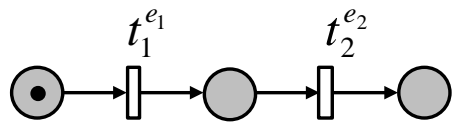

Figure 5. PN representing the sequence $\mathrm{e}_{1} \mathrm{e}_{2}$

Until $8^{\text {th }} \mathrm{I} / \mathrm{O}$ vector, the situation is quite similar: every new event is processed and the model is updated.

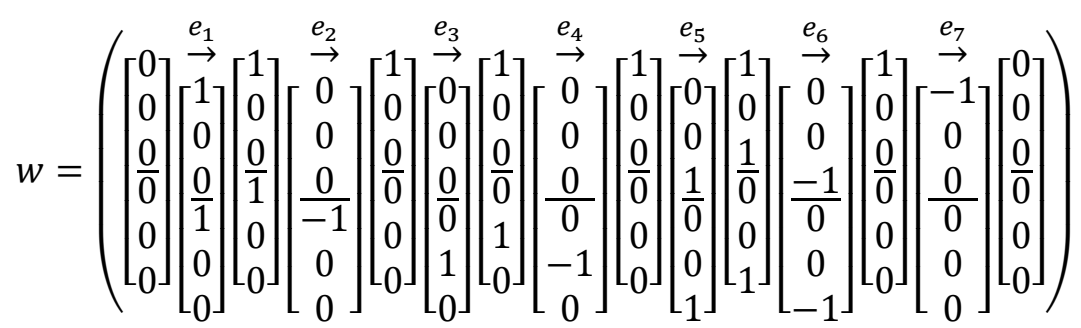

When the $9^{\text {th }}$ vector $w(9)=\left[\begin{array}{lllllll}1 & 0 & 0 & \mid & 1 & 0 & 0\end{array}\right]^{T}$ is processed, the event $\tau(8)=e_{1}=$ $\left[\begin{array}{lllllll}1 & 0 & 0 & \mid & 1 & 0 & 0\end{array}\right]^{T}$ is computed and the trace $\tau^{1}(8)$ is identified through Step 3 as an already observed trace $e_{1}$. Since it leads to the same marking than the input place of $t_{1}^{e_{1}}$, such a place and the output place of $t_{7}^{e_{7}}$ can be merged as shown in Figure 6.

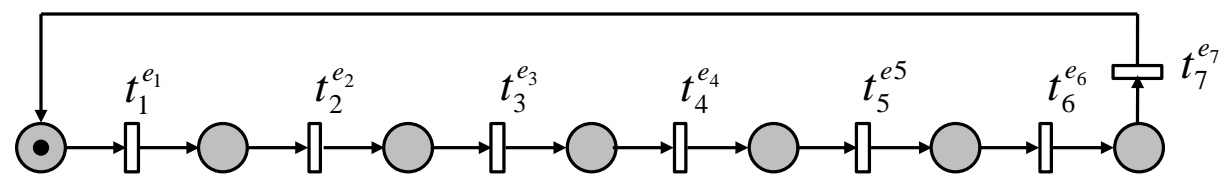

Figure 6. Internal model for the first detected cycle

Since a cycle is found, steps 4 and 5 of the algorithm are executed, leading to an intermediate IPN model shown in Figure 7.

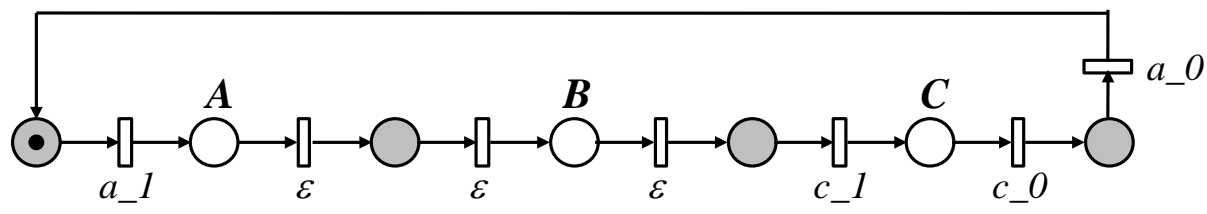

Figure 7. IPN for the first detected cycle

Simultaneously to the creation of the intermediate IPN, more I/O vectors are added to the observed sequence and the $\mathrm{PN}$ is updated: 


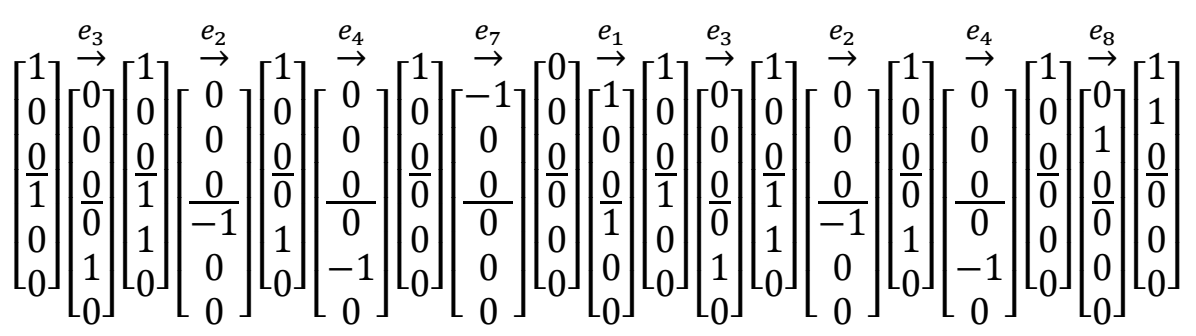

Two more cycles are found in this sequence and intermediate IPN models are created. We show only the PN obtained after finding the second cycle (Figure 8) and its equivalent model transformed by analysing concurrency (Figure 9). After applying the steps 4 and 5 the IPN obtained from this PN is shown in Figure 2.

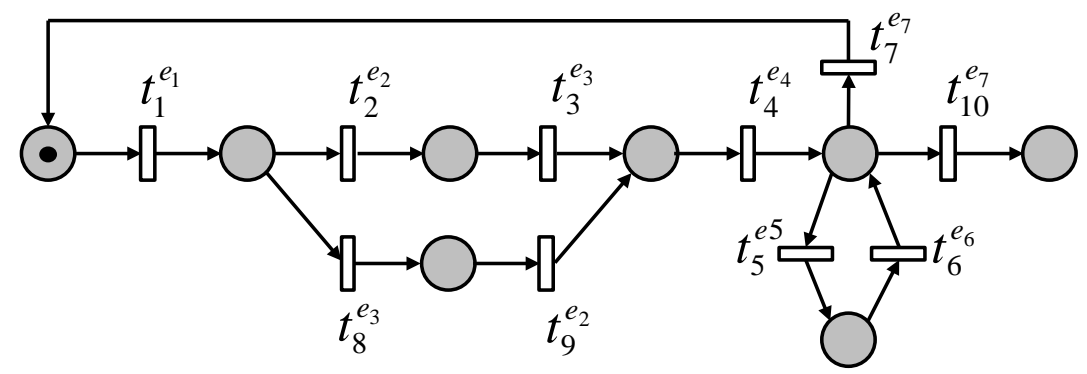

Figure 8. PN corresponding to the whole $\mathrm{I} / \mathrm{O}$ sequence

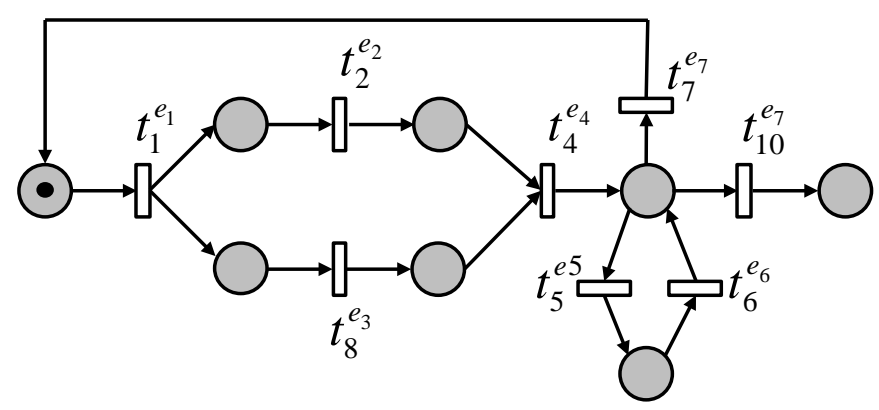

Figure 9. Equivalent internal model representing concurrency

Remark. The simplification by analysis of concurrency in Step 4 is not strictly necessary for representing the event vector sequences; however the equivalent model with concurrent transitions may be simpler. The aim of this simplification is not minimizing the number of nodes in the model, but obtaining fairly reduced models useful for clearly express the DES behaviour.

\section{Method implementation and application}

\subsection{An identification tool}

Based on the algorithms presented in section 3, a software tool has been developed to automate the IPN model identification. The architecture of the tool is shown in Figure 10.

The user interface allows capturing the input/output sequence and displays the identified model graphically. Several data are provided to the tool: a text file containing the I/O sequence (with one line per $\mathrm{I} / \mathrm{O}$ vector), the parameter $\kappa$ (which is fixed by the user), the names (mnemonics) of the input and output signals, and the desired name of output file. Additionally it is specified the order in which inputs and outputs appear in the txt file (since due to data collection issues they could be inverted) and the index numbers of the signals to take into account if a mask is going to be applied. 
Later, an input reader component processes the input file and transforms the input/output sequence into a vector sequence. These vectors will be delivered to a component called "Algorithm" in which the identification algorithm is implemented. The output of this component is a dot file that can be given to the Graph Visualization Software (Graphviz, 2011) to generate an image file jpg.

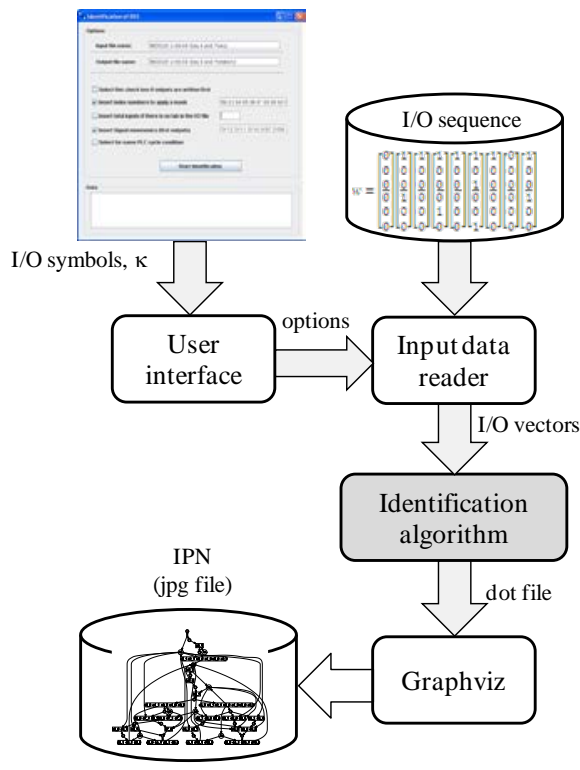

Figure 10. Software architecture

The presented identification tool has been successfully tested on several examples of diverse size and complexity. Below, for more of clarity, we illustrate the use of such software tool through a small size case study.

\subsection{Case study}

For space considerations a small size application example is presented in this paper dealing with an automated manufacturing system; it is taken from (Roth, 2009). The purpose of such a system is to sort parcels according to their size (Figure 11). It has 9 input signals from sensors of the plant: $\mathrm{a}_{0}$, $a_{1}, a_{2}, b_{0}, b_{1}, c_{0}, c_{1}, k_{1}, k_{2}$, and 4 outputs signals to the actuators: $A^{+}, A^{-}, B, C$.

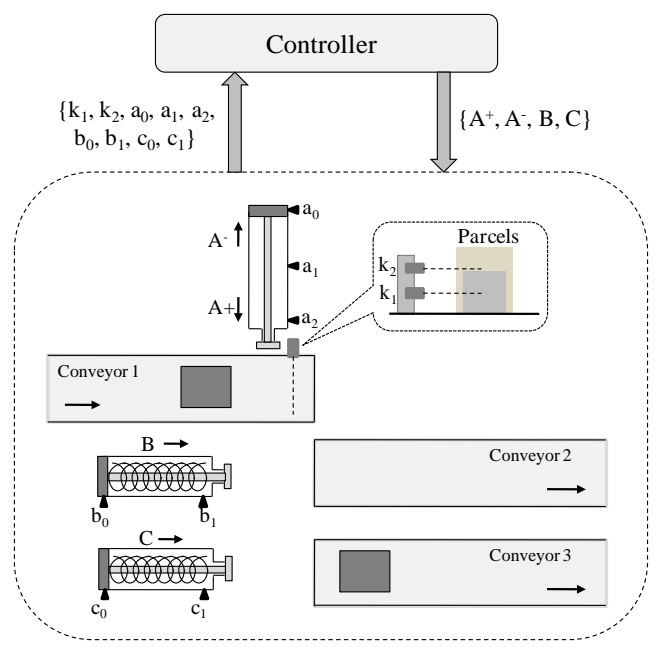

Figure 11. Layout of the system case study 
A short sequence consisting of $216 \mathrm{I} / \mathrm{O}$ vectors has been processed; it is shown on Figure 12. Notice that even if this system operates repetitively, cycles are not specified in the sequence and they must be identified. Binary values of each $I / O$ vector correspond to signals $A^{+}, A^{-}, B, C, k_{1}, k_{2}, a 0, a 1, a 2, b_{0}, b_{1}, c_{0}$, and $c_{1}$ respectively. Notice that the input and output signals order in each vector is inverted (first the outputs, later the inputs). The identification parameter for this case study is $\kappa=1$.

In a first stage the identified model based on the first $31 \mathrm{I} / \mathrm{O}$ processed vectors is shown in Figure 13. It can be noticed that cylinder $C$ has not worked yet, since big parcels have not yet arrived.

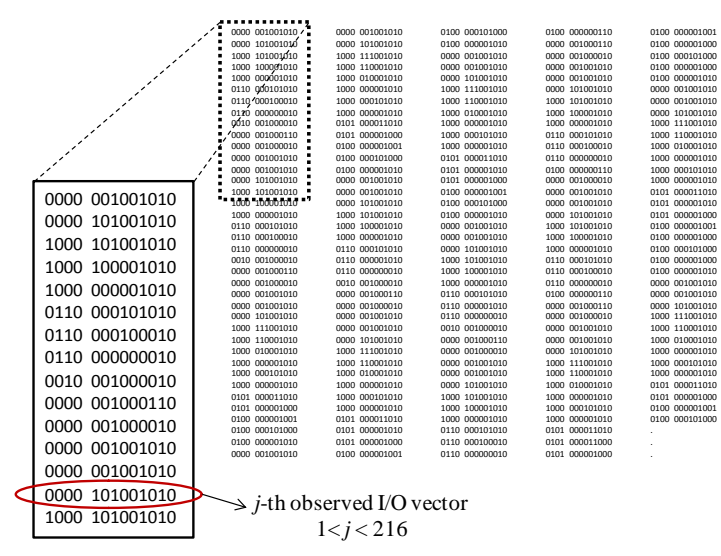

Figure 12. I/O sequences of the case study

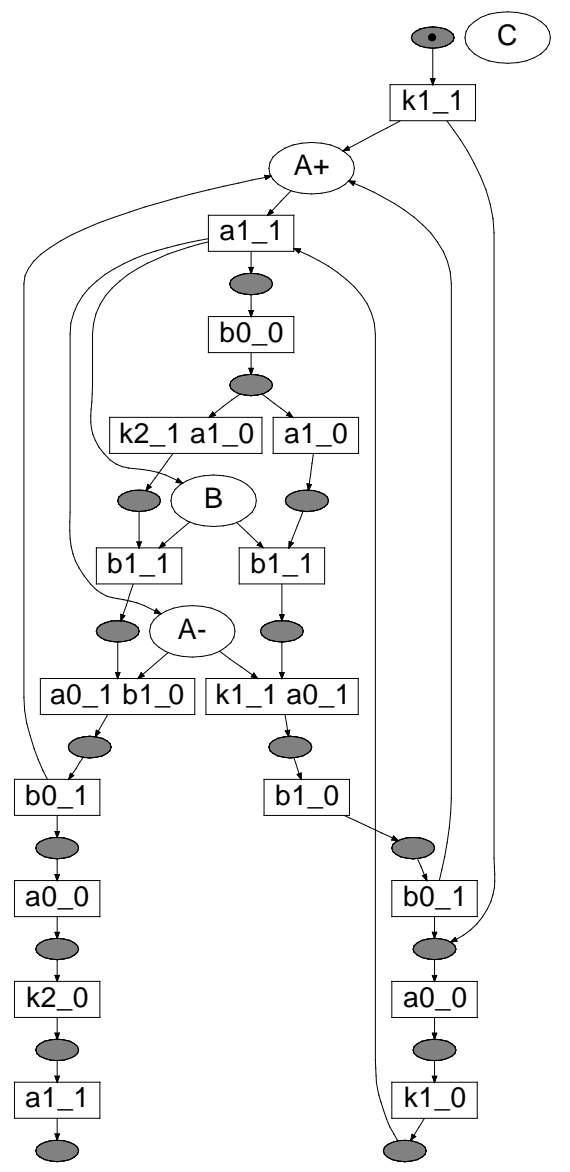

Figure 13. Obtained model after processing $31 \mathrm{I} / \mathrm{O}$ vectors

Using the complete sequence of I/O vectors the obtained model is shown in Figure 14. The identification procedure finds successfully cyclic behaviour in the single sequence of I/O vectors. The processing times for obtaining the modes of figures 13 and 14 are 31 and 47 ms respectively; the test were performed using a PC based on an Intel Core 2 Duo E6850 processor at $3.00 \mathrm{GHz}$ with 2.00 GB of RAM under Windows XP Professional 2002 Service Pack 2.

4.3 Further simplification

Notice that in this IPN model there are paths formed by several non observable places. This is due to the observation of input changes that do not affect the outputs, but that changes the system internal state. In order to obtain a more compact model a simplification strategy presented in (Estrada 2010a) has been applied; it is recalled below. It consists in merging several places, representing internal behaviour whose detected events do not have effect on the outputs, into a single one where an output event occurs. Consider the following I/O vector sequence involving one input $x$ and two outputs $A, B$ :

$$
\left[\begin{array}{c}
x \\
A \\
B
\end{array}\right] \quad\left[\begin{array}{l}
0 \\
1 \\
0
\end{array}\right]\left[\begin{array}{l}
\frac{1}{1} \\
0
\end{array}\right]\left[\begin{array}{l}
\frac{1}{0} \\
0 \\
1
\end{array}\right]
$$


This sequence can be represented as: $A \stackrel{x_{-} 1}{\longrightarrow} A \stackrel{\varepsilon}{\longrightarrow} B$, which can be compacted as: $A \stackrel{x_{-} 1}{\longrightarrow} B$. This can be generalized to: $A \stackrel{e_{i}}{\longrightarrow} A \stackrel{e_{j}}{\longrightarrow} \ldots A \stackrel{e_{k}}{\longrightarrow} B \cong A \stackrel{e_{i} e_{j} \ldots e_{k}}{\longrightarrow} B$

The application of this simplification procedure, also included in the software tool yields the IPN model shown in Figure 15. The execution time including the compacting procedure for obtaining this model is 78 ms.

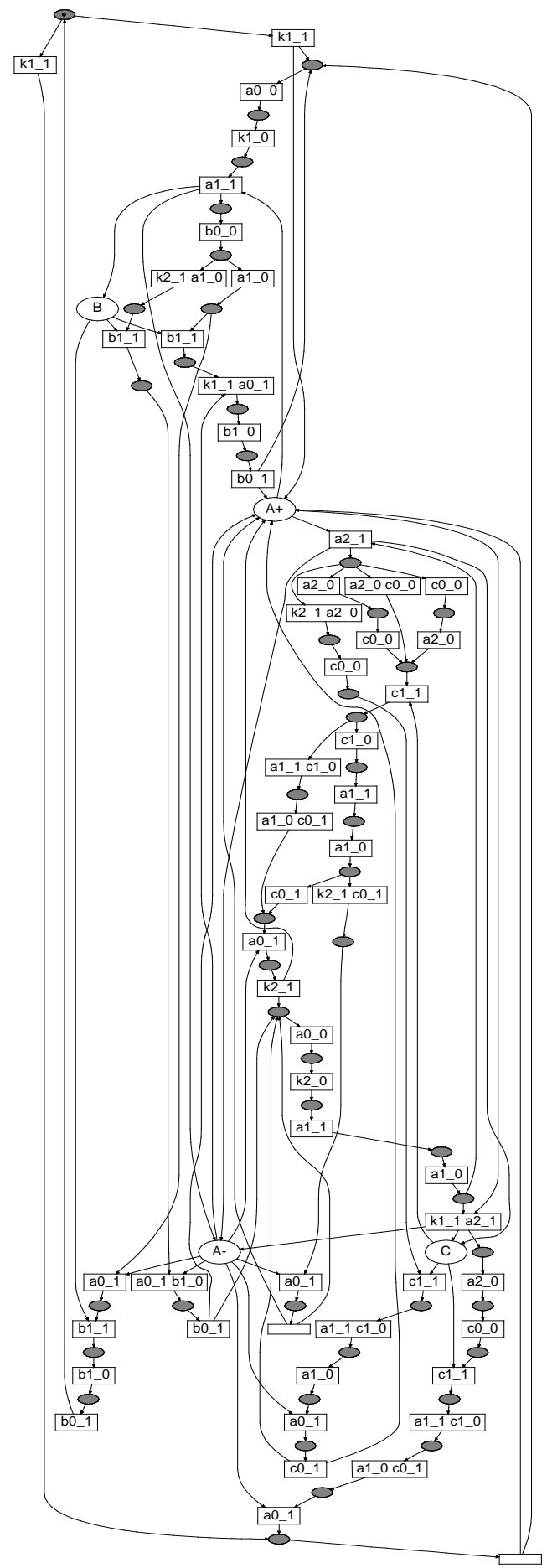

Figure 14. IPN for the case study 


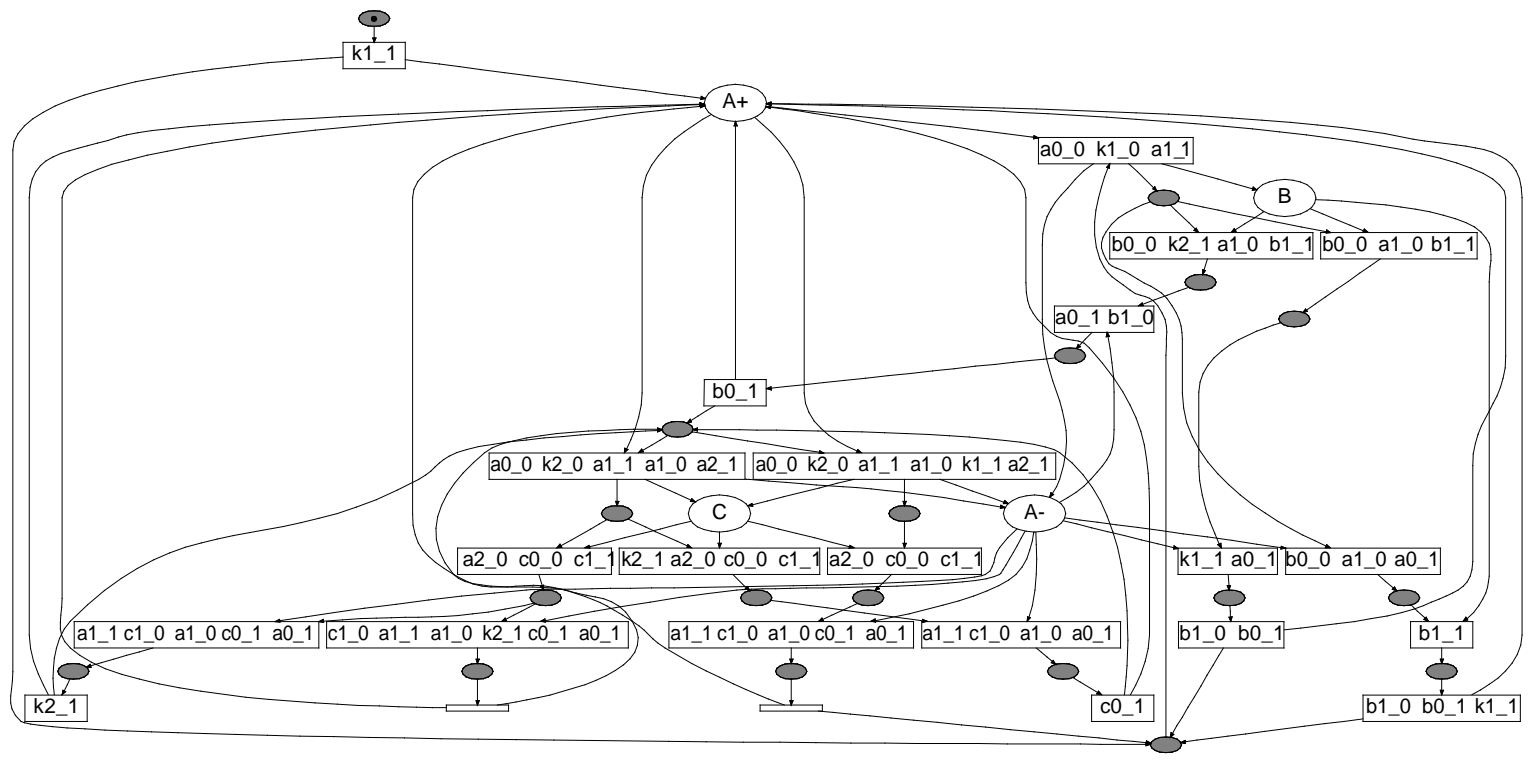

Figure 15. IPN model after applying the simplification rule

4.4 The role of the parameter $\kappa$

The parameter $\kappa$ helps to distinguish sequences of events during the construction of the basic internal model; its value indicates the history of past $\kappa$ events that have to be considered for deciding the state equivalence. When high values of $\kappa$ are used, it is possible to distinguish more sequences avoiding path fusion during the model construction; thus the obtained models are more accurate but less compact. Thus, low values of $\kappa$ allow more state fusions; the obtained model are more compact but more paths can be created yielding an overrepresentation of the observed behaviour.

In order to illustrate the influence of $\kappa$ in the identification procedure, Figures 16 and 17 show the IPN model and the simplified model, respectively obtained from the same $\mathrm{I} / \mathrm{O}$ sequence when $\kappa=2$ is used; the processing time are $125 \mathrm{~ms}$ and $140 \mathrm{~ms}$ respectively. It can be noticed that these models are less compact than that obtained with $\kappa=1$.

In general it is not possible to fix a priori the value of $\kappa$, since it is assumed that the system is unknown. However, in practice the identification procedure can be applied using several values of $\kappa$ (because it is not time consuming) until there are no significant variations on the number of nodes. Compact models allow a first approximation to the understanding of the system functioning, whilst larger models provide a more precise description. For small examples, such as that included in this section, $\kappa=1$ allows distinguishing event sequences whilst compact models are built. 


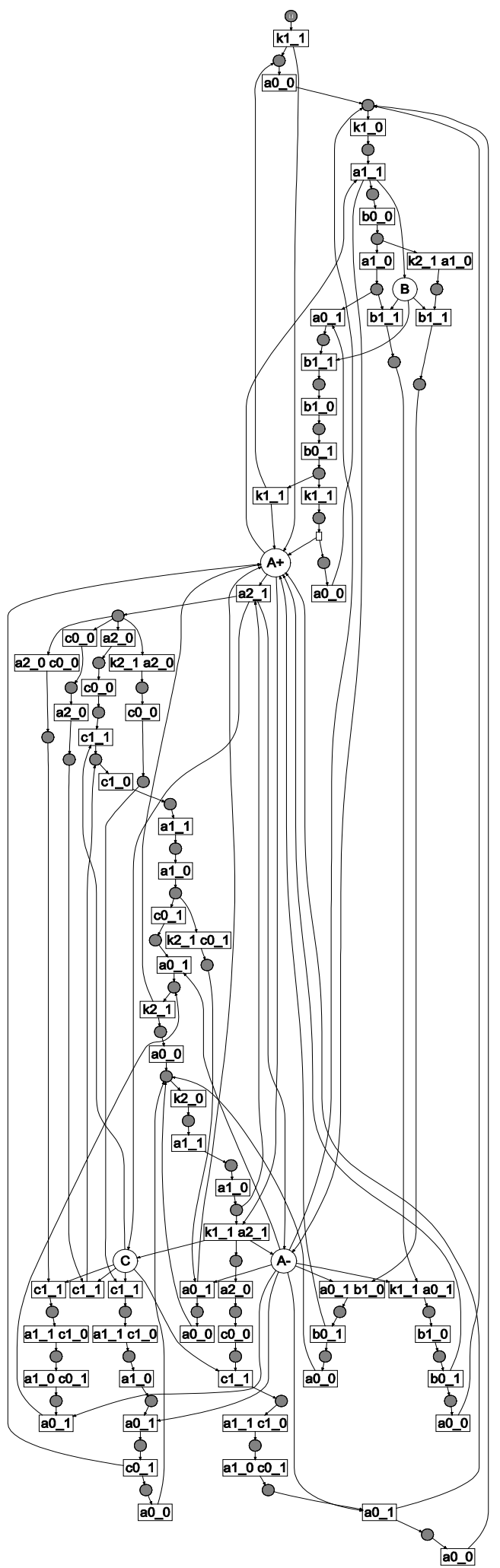

Figure 16. IPN obtained using $\kappa=2$

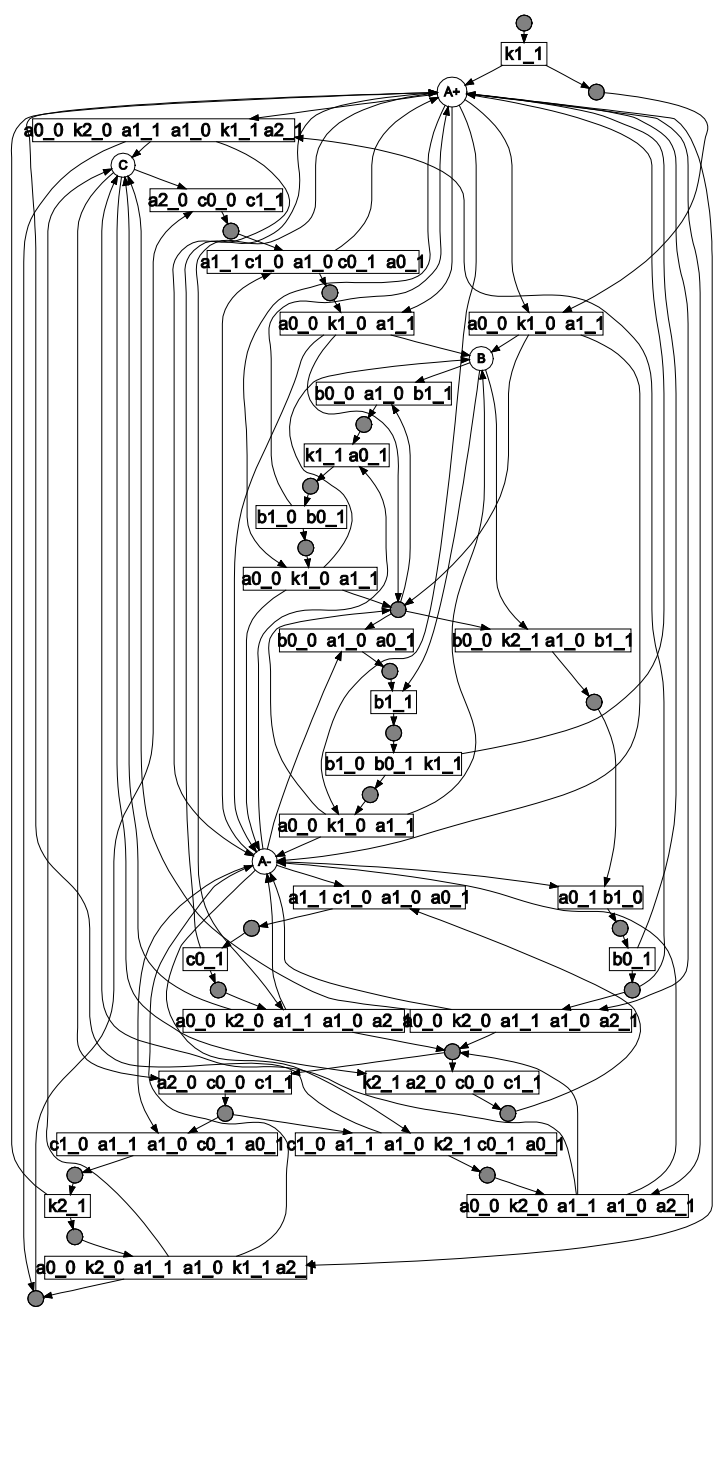

Figure 17. Simplified IPN obtained using $\kappa=2$ 


\section{Concluding remarks}

Stepwise identification of automated manufacturing systems has been addressed. This black-box approach allows obtaining IPN models from a single input/output sequence that exhibits the closed loop behaviour of PLC-based controlled plants. The proposed technique builds progressively the IPN from an I/O vector sequence collected from the system during its operation and stored into a data base. In case of new vectors are recorded, this data can be processed to update the model already built. The identified model is a close approximation of the compound controller-plant behaviour, which can for instance be detailed for performance evaluation, controller redesign or model-based diagnosis purposes.

The approach herein presented allows dealing with real complex automated DES because it takes into account technological characteristics of actual controlled systems, and because it is based on efficient algorithms (whose computational complexity is polynomial-time).

Furthermore, several features of our method have not been addressed in current literature on the matter. Although in (Meda, 2001, 2003, 2005) cycle finding from single sequence is dealt, it is based on the observation of the same initially observed outputs vector, which is not realistic for real systems; besides system's inputs are not taken into account. In (Klein, 2005), (Roth, 2009, 2010) are considered both inputs and outputs, but cyclic sequences are supposed to be known. Techniques in (Giua 2005), (Cabasino, 2007), (Dotoli, 2011), (Cook, 2004)(van der Aalst, 2004), (Ould El Medhi, 2006, 2012) process as input data a language generated by the system, which is given as events sequences, regardless how the events are obtained from I/O data. Regarding our previous works (Estrada 2009), (Estrada 2010), the assumption about cyclic sequences as input data is dropped off in the current proposal, allowing addressing actual controlled DES.

Current research focuses on the reduction of the obtained model by the analysis of the ulterior influence of inputs that apparently do not provoke changes in the outputs. Also, the inference of non observed behaviour regarding concurrent sub-processes has to be studied in depth to obtain more compact and expressive models.

\section{Acknowledgement}

The first author is sponsored by CONACYT Mexico, Grant number 50312 and Région Ile de France.

\section{References}

Angluin, D., 1988. Queries and Concept Learning, Machine Learning, Vol. 2, No.4, 319-342

Cabasino, M. P., Giua, A., Seatzu, C., 2006. Identification of unbounded Petri nets from their coverability graph, Proc. of the IEEE Int. Conf. on Decision and Control, San Diego, CA, USA, 434 - 440

Cabasino, M.P., Giua, A., Seatzu, C., 2007. Identification of Petri Nets from Knowledge of Their Language, Discrete Event Dynamic Systems, Vol. 17, No. 4, 447-474

Cook, J. E., Du, Z., Liu, C., Wolf, A., 2004. Discovering models of behavior for concurrent workflows, Computers in Industry, Vol. 53, No. 3, 297 - 319, doi: 10.1016/j.compind.2003.10.005

DiCesare, F., Harhalakis, G., Proth, J. M., Silva, M. and Vernadat, F. B., 1993. Practice of Petri Nets in Manufacturing. Chapman and Hall.

Dotoli, M., Fanti, M. P., Mangini, A. M., 2008. Real time identification of discrete event systems using Petri nets, Automatica, Vol. 44, No. 5, 1209-1219

Dotoli, M., Fanti, M. P., Mangini, A. M., Ukovich, W., 2011. Identification of the unobservable behaviour of industrial automation systems by Petri nets, Control Engineering Practice, Vol. 19, No. 9, 958-966

Estrada-Vargas, A.P., Lesage, J.-J., López-Mellado, E., 2011. Stepwise Identification of Automated Discrete Manufacturing Systems, IEEE Int. Conf. on Emerging Technologies and Factory Automation, Toulouse, France, USB Paper N4-6, 8p 
Estrada-Vargas, A.P., López-Mellado, E., Lesage, J.-J., 2009. Off-line Identification of Concurrent Discrete Event Systems Exhibiting Cyclic Behaviour, Proc. of the IEEE Int. Conf. on Systems Man and Cybernetics, San Antonio, TX, USA, 181-186

Estrada-Vargas, A.P., López-Mellado, E., Lesage, J.-J., 2010. An Identification Method for PLC-based Automated Discrete Event Systems, Proc. of the IEEE Int. Conf. on Decision and Control, Atlanta, USA, 6740-6746

Estrada-Vargas, A.P., López-Mellado, E.. Lesage, J.-J., 2010. A Comparative Analysis of Recent Identification Approaches for Discrete-Event Systems, Mathematical Problems in Engineering. Volume 2010, Hindawi. doi:10.1155/2010/453254

Fanti, M. P., Seatzu, C., 2008. Fault diagnosis and identification of discrete event systems using Petri nets, Proc. of the 9th Int. Workshop on Discrete Event Systems, Göteborg, Sweden, 432-435

Giua, A., Seatzu, C.,2005. Identification of free-labeled Petri nets via integer programming, Proc. of the IEEE Conf. on Decision and Control and the European Control Conf, Seville, Spain, 7639 - 7644

Gold, E.M., 1967. Language Identification in the Limit, Information and Control, Vol. 10, No.5, 447-474

Gradišar, D. , Mušič, G., 2007. Production-process modelling based on production-management data: a Petrinet approach International Journal of Computer Integrated Manufacturing Vol. 20, No. 8, 794-810

Graphviz, 2011. http://www.graphviz.org/

Hiraishi, K., 1992. Construction of Safe Petri Nets by Presenting Firing Sequences, Lectures Notes in Computer Sciences, Vol. 616, 244-262

Jeng M., Xie, X., 2005. Discrete event system techniques for CIM Special Issue. International Journal of Computer Integrated Manufacturing. Vol. 18, Issue 2-3.

Kella, J., 1971, Sequential Machine Identification, IEEE Trans. on Computers, Vol. 20, No. 3, 332-338

Klein, S., Litz, L., Lesage, J.-J., 2005. Fault detection of Discrete Event Systems using an identification approach, 16th IFAC World Congress, CDROM paper n02643, 6 pages, Praha (Czech Republic)

Lanubile, F., Visaggio, G., 2002. Iterative Re-engineering to compensate for Quick-Fix Maintenance, Proc. Of the IEEE Int. Conf. on Software maintenance, Opio, France, 140-146

Meda-Campaña, M., López-Mellado, E., 2001. A passive method for on-line identification of discrete event systems, Proc. of the IEEE Int. Conf. on Decision and Control, Orlando, Florida, USA. 4990-4995

Meda-Campaña, M., López-Mellado, E., 2003. Required Transition Sequences for DES identification, Proc. of the IEEE Conf. on Decision and Control, Maui, Hawaii, USA, 3778-3782

Meda-Campaña, M., López-Mellado, E., 2005. Identification of Concurrent Discrete Event Systems Using Petri Nets, Proc. of the IMACS 2005 World Congress, Paris, France, 1-7

Ould El Medhi, S., Bekrar, R., Messai, N., Leclercq, E., Lefebvre, D., Riera, B., 2012. Design and identifcation of stochastic and deterministic stochastic Petri nets, IEEE Trans. on Systems, Man and Cybernetics - Part A, Vol. 42, No. 4, 931 - 946, doi: 10.1109/TSMCA.2011.2173798

Ould El Medhi, S., Leclercq, E., Lefebvre, D., 2006. Petri nets design and identification for the diagnosis of discrete event systems, IAR Annual Meeting, Nancy, France

Richetin, M., Naranjo, M., Luneau, P., 1984. Identification of Automata by Sequential Learning, Pattern Recognition Letters 2, Vol. 2, No. 6, 379-385

Roth, M., Lesage, J.-J., Litz, L., 2009. An FDI Method for Manufacturing Systems Based on an Identified Model, Proc. of IFAC Symposium on Information Control Problems in Manufacturing (INCOM 2009), Moscow, Russia, 1389-1394

Roth, M., Lesage, J.-J., Litz, L., 2010. Black-box identification of discrete event systems with optimal partitioning of concurrent subsystems, Proc. of the American Control Conf. (ACC 2010), Baltimore, Maryland, USA, 2601-2606

Van der Aalst, W., Weijters, T., Maruster, L., 2004. Workflow Mining: Discovering Process Models from Event Logs, IEEE Trans. on Knowledge and Data Engineering, Vol. 16, No. 9, 1128-1142 